\title{
Position Paper: Why Intermittent Computing Could Unlock Low-Power Visible Light Communication
}

\author{
James Scott Broadhead \\ J.S.Broadhead@tudelft.nl \\ Delft University of Technology \\ Delft, Netherlands
}

\author{
Przemysław Pawełczak \\ P.Pawelczak@tudelft.nl \\ Delft University of Technology \\ Delft, Netherlands
}

\begin{abstract}
Intermittent computing could be combined with low-powered visible light communication systems to facilitate novel sensor networks. This position paper provides a framework for future research by highlighting synergies between the technologies, and explores how techniques developed in intermittently-powered systems can be used to provide enhanced stability to ultra-low power visible light communication.
\end{abstract}

\section{CCS CONCEPTS}

- General and reference $\rightarrow$ Surveys and overviews; • Computer systems organization $\rightarrow$ Sensors and actuators.

\section{INTRODUCTION}

Realising the vision of Internet of Things (IoT) held by the systems community will not only require significant technological innovation, but also a re-evaluation of advancements that have already been made. This paper looks to provoke future studies on the IoT by considering the union of two distinct fields: intermittent computing [22] and visible light communication [25]. Whilst both have been considered independently, we believe that it is prudent to position them as symbiotic in their ability to further enhance the IoT, and that by combining the technologies a new genre of devices can be developed.

As the number of connected devices continues to grow (an estimated 29.3 billion by 2023 [7]) so too will the number of batteriesa fact that has raised concern in the research community. This seemingly unsustainable growth may have significant environmental consequences [8], impose limits on the minimum device sizes achievable, and cause issues when batteries need to be replaced [13]. The pressing need for alternatives has encouraged the community to look for novel 'battery-free' intermittently powered systemsthus spawning the field of 'Intermittent Computing' [22]. The field looks to provide hardware/software to enable programs to successfully execute under significant power failures by (for example) frequently checkpointing the system state to non-volatile memory and restoring to volatile memory when sufficient energy is available. Of course, this shift towards battery-free operation injects an inherent volatility; creating a number of challenges, not least the networking of devices [22]. Facilitating the development of intermittent sensor networks (through mathematical and computational

(C)2020 Association for Computing Machinery.

This is the author's version of the work. It is posted here for your personal use. Not for redistribution. The definitive Version of Record was published in Light Up the IoT (LIOT '20), September 21, 2020, London, United Kingdom, https://doi.org/10.1145/3412449.3412546. models), and beginning to design them in practice, will be critical to IoT systems of the future.

Communication through visible light has been a fixture in our societies for centuries [26], however its relevance to the IoT, and the use of low-powered light emitting diodes (LEDs) to transfer data, is a much more recent phenomenon. In itself, Visible Light Communication (VLC) also presents a number of potential challenges. Active VLC is very energy expensive considering environmental constraints. Given this, an increasing amount of work has been put into designing low-powered VLC systems [20,28]. The advent of passive VLC [31] (modulating light already present in an environment) is another area of research that looks to reduce energy consumption.

The idea of battery-free operation in VLC is a highly contemporary one within the research community, with three recent publications demonstrating this concept. The work of [21] presents an energy harvesting VLC system design that functions in radio frequency hostile work environments, where hard-wiring devices and replacing batteries is not feasible. Here the combination of VLC and energy harvesting overcomes the environmental challenges presented, providing a clear use case of combining the technology. Whilst the system has the hallmarks of an intermittent device, indeed the power supplied is intermittent, the system does not implement intermittently operating strategies (such as energy-aware system state checkpointing) to enhance performance. A clear extension of this work would be the inclusion of such a regime-the system hardware is also much larger than would be expected for widespread deployment, so miniaturisation would also be advantageous. Another piece of work, from a different set of authors [12] considers a combined VLC and radio frequency (RF) front-end with an RF backscatter system to enable battery-free communication. The system does not consider intermittently operating strategies as part of its design, however does set an interesting precedent for the integration of VLC with low-power radio to overcome deployment challenges. Finally, the work of [19] demonstrates a battery-free passive VLC architecture that clearly aligns with the vision of lowpower VLC presented in this paper. Whilst the system does not consider the deployment of checkpointing, it does provide a good springboard from which this paper has manifested.

Whilst much work has been done, it is clear that significant room still exists for a re-evaluation of how existing systems can be improved and for new battery-free intermittently-operating VLC devices to be created. This paper looks to set a vision for future battery-free sensor networks by presenting scenarios in which strategies developed for intermittent computing and VLC would be mutually beneficial. 


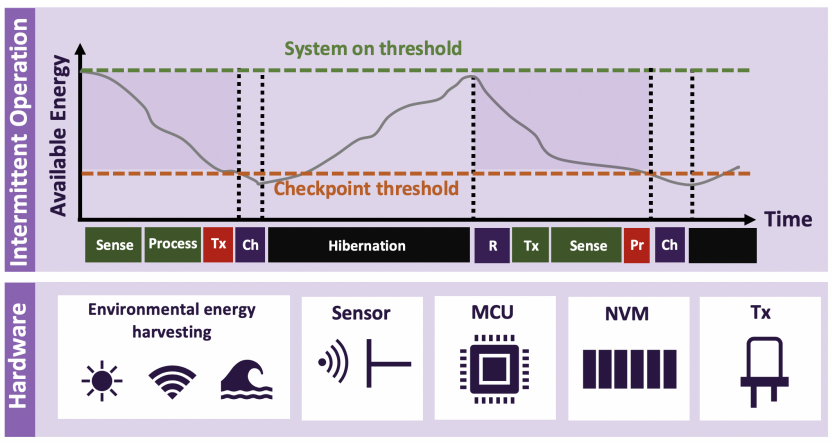

Figure 1: Proposed intermittent VLC system architecture. Here the overlay of an energy-aware checkpointing (Ch) strategy will improve the performance of the systems since power failure will not necessitate a full cycle of sensing, processing, and transmission. In the figure, the first transmission ( $\mathrm{Tx})$ is disrupted, however due to checkpointing $(\mathrm{Ch})$ the data is saved and the state of the system is efficiently restored (state $R$ ), rather than starting from scratch. Pr represents disrupted processing.

The rest of the paper is structured as follows. Section 2 presents a vision statement of the necessary hardware and software strategies that should be deployed to enhance system functionality. Section 3 considers research opportunities in the battery-free intermittentlypowered VLC field given an assessment of notable challenges. Then, Section 4 highlights several key areas where an integrated VLC/intermittently-powered systems would supersede traditional battery-powered or RF-communicating devices. Finally, the paper concludes in Section 5.

\section{INTERMITTENTLY-POWERED VLC}

The combination of VLC and intermittent operation is not a silver bullet for all sensor networks. However transient computing techniques could provide a useful support structure to ultra-low powered battery-free VLC systems, and thus allow them to fulfil a number of niche applications. Being able to successfully execute programs, even with high frequency power failure, would provide an extra level of stability. We feel that strategies guaranteeing computation despite frequent power failures should be ingrained into the ethos of low-power VLC design.

\subsection{Intermittently-powered VLC Architecture}

Traditional energy-neutral computing has looked to process jobs 'as normal' by storing collected energy in batteries, thus mitigating power failure. The field of intermittent computing instead seeks to accept failure as an inevitable part of the system, and uses software strategies (such as checkpointing) to restore the system's state, from the last moment of power failure, when sufficient energy becomes available. Intermittent operation may be valuable to current low-powered VLC systems as it will improve performance under extreme power constraints, therefore enabling smaller device footprints (for a given level of performance) and allowing systems to work in harsher environments. In practice we can visualise our system as given in Fig. 1. The hardware architecture of such a device is not necessarily unique (an equivalent systems could be found in [21] and the work of [12] presents battery-free hardware with a VLC front-end), however the overlay of software developed in the intermittent computing domain to this hardware is a novel addition.

2.1.1 System Hardware. Any co-deployment of the two technologies will share a number of fundamental hardware components listed below.

- Energy harvesting: By definition the device will be energy harvesting so the system must be able to source energy from its environment (be this through solar, mechanical movement, radio etc.). For instance, the work of [21] deploys a Powercast P21XXCSR-EVB energy harvesting board which converts RF energy in the environment into DC power, which is then stored in a super-capacitor.

- Sensing: The devices will also act to sense target data from their environment. Practical measurements could include temperature, $\mathrm{pH}$, oxygen levels and will allow users to continuously monitor environmental conditions.

- Processing: The MCU will undertake processing of the data (such as filtering) before it is relayed for transmission. The popular Texas Instruments MSP430 MCU is likely to prove a suitable candidate for initial designs due to its energy efficiency, as already used for instance by $[19,21]$.

- Computation storage: The non-volatile memory (NVM) (most likely FRAM in current system designs) acts to preserve processing data in the event of a power failure. A save to the NVM would occur when a lower energy threshold is reached (as can be seen in Fig. 1).

- Transmission: Once the data has been collected and processed, it will then be forwarded to an appropriate monitor through the transmitter of the device. This will either be a low-powered LED or a LCD/smart surface able to modulate light (as is the case in PassiveVLC [31] or RetroVLC [19]). The work of [21] uses a SSL-LX100133XUWC LUMEX standard LED to forward data, whilst [12] instead deploys an RF backscatter modulator for uplink. The work of [19] uses a retro-reflector fabric that backscatters light with an LCD shutter to then modulate the reflected light.

Compact comparison of VLC battery-free technologies is also provided in Table 1.

2.1.2 System Software. The novelty in our proposed device is the porting of strategies to preserve computation despite power interrupts to the battery-free energy harvesting VLC architecture of Section 2.1.1. Our system would utilise transient operating systems (such as [3]) that deploy systematic checkpointing to force hibernation when available energy is low. Here, data currently being processed (such as data filtering) is saved to the non-volatile memory before a system power failure and then rapidly restored to the processing location after a sufficient threshold energy becomes available.

By implementing strategies from the intermittent computing domain, the system will naturally be more resilient to power failure, since processing costs will be minimised (here a system power 
Table 1: Comparison of low-powered battery free devices, externally low-powered VLC [ $\dagger]$, and battery-free non-VLC $[\ddagger]$ for comparison

\begin{tabular}{|c|c|c|c|c|c|c|c|c|}
\hline Device & Size & Comm. range $(\mathrm{m})$ & Uplink (Kbps) & MCU & Fabrication & Harvester & Capacitor & Scenario \\
\hline Liu et al. [21] & $\approx 20 \times 20 \mathrm{~cm}$ & 0.14 & 100 & MSP430FR5969 & Evaulation board & P21XXCSR-EVB (RF) & $50 \mathrm{mF}$ & Indoors \\
\hline EDISON [12] & Not given & 20 & 2.9 & MSP430FR5969 & Dedicated PCB & $\begin{array}{l}\text { Solar panel } \\
\text { (MP3-37) }\end{array}$ & $100 \mu \mathrm{F}-6.8 \mathrm{mF}$ & Indoors/Outdoors \\
\hline RetroVLC ViTag [19] & $8.2 \times 5.2 \mathrm{~cm}$ & 2.4 & 0.5 & MSP430G & Dedicated PCB & Solar panel & Not given & Indoors \\
\hline PassiveVLC ViTag [31] & "Credit card" & 4.5 & 1 & MSP430 & Dedicated PCB & Solar panel & Not given & Indoors/Outdoors \\
\hline † OpenVLC [29] & "Credit card" & 1 & 2 & TI AM3359 CPU & Beagle Bone Black [11] & $\mathrm{N} / \mathrm{A}$ & $\mathrm{N} / \mathrm{A}$ & N/A \\
\hline \# Cinamin [5] [Theoretical] & $5 \times 5 \times 3.4 \mathrm{~mm}$ & $1-3$ & N/A & N/A & Dedicated PCB & Solar panel & Not given & Indoors \\
\hline \#Botoks $[9]$ & $2.5 \times 2.5 \mathrm{~cm}$ & $10-100$ & $50-200$ & MSP430FR5994 & Dedicated PCB & Solar panel & $100 \mu \mathrm{F}$ & Indoors \\
\hline
\end{tabular}

failure will not delete partially processed sensor data, but rather temporarily halt the process). This addition would enhance work currently seen in literature and would increase resilience in energy constrained environments. The transient operating strategies developed in [3] and [16] show clear energy and performance improvements over the duration of a program execution compared to systems that do not implement checkpointing.

\section{RESEARCH DIRECTIONS}

It is clear from a systems perspective that significant effort should be invested into practically designing platforms that integrate state preservation strategies into existing low-powered VLC. This will then extend to the development of novel systems (specifically in the areas highlighted in Section 4).

It is difficult to properly guide future research without also addressing the challenges that we are attempting to overcome. Batteryfree VLC platforms and transient operation present a number of obstacles, not least due to the infancy of both fields. If we are to design connected VLC systems that utilise energy harvesting and intermittent operation, we must also begin to understand to what extent theoretical challenges will limit practical success. An academically lucrative area of research will be in mathematically and computationally modeling VLC/intermittent systems, therefore providing useful insight to system designers.

\subsection{Designing the Communication Stack for Intermittent VLC}

3.1.1 Challenge. One of the key bellwethers for the success of intermittently operating VLC will be in designing an appropriate communication stack. The ability to perform communication in intermittent devices is fundamentally hampered by the unknown period of off time. The associated energy cost for communication is also significant, so the intermittently operating device must be tactful in when it assigns energy to the forwarding of data [22] Effective synchronisation of intermittent devices is also an active area of work and a clear challenge to researchers.

The work of [27], which presents an LED-LED VLC network, makes the case that the efficiency of the synchronisation protocols is critical to the overall throughput of the links. The paper presents a simple VLC physical layer (PHY) and a contention based VLC medium access control (MAC) protocol as a means of providing communication between the LEDs. The synchronisation of the devices is a key part in facilitating successful operation.

The overarching challenge of communication between intermittently powered VLC devices is the inherent volatility of the receiver.
If a device is powered and wants to forward information, it has very little knowledge of the state of the receiving device (whether it is hibernating or not) so may communicate blindly. Traditional solutions, such as acknowledgements, may be too costly given the restricted energy budget. Active listening of the receiver could also incur a unacceptable cost.

3.1.2 Research Opportunity. Given these challenges, we are presented with a clear research opportunity: to understand how the loss of synchronisation in intermittent devices will affect communication. Since the synchronisation of intermittent platforms is still an open area of research, and synchronisation has been identified in areas of literature as an important part of VLC communication [27], much work will either be needed in designing light PHY and MAC protocols that effectively account for the potential loss of synchronisation due to unpredictable intermittent operation, or in providing synchronisation across intermittent devices.

It may be interesting to also consider energy-aware strategies to update other connected devices of hibernation (i.e. a device could notify local devices of its power status) although the energy impact of this would need to be assessed.

\subsection{Networking Devices}

3.2.1 Challenge. As is succinctly presented in [22], and eluded to in Section 3.1, one of the most relevant challenges of intermittent computing is networking-this will continue to cause concern in an intermittently operating VLC system. Being able to successfully transfer sensor data through a low-powered and frequently inactive network is an open problem in research. This is compounded by the fact that devices collect energy from their environment, so neighbouring devices are likely to be subjected to similar environmental conditions (meaning that power failure may affect entire network regions). Reliable timekeeping has also been explored as current challenges facing intermittent communication and the construction of networks [9]. If we are to transfer useful information there must be some agreed uniformity between devices. The hibernation of systems presents clear theoretical and practical issues for system designers. Ad-hoc and multi-hop networks would also need to make decisions about whether to collect data or forward data from other devices.

3.2.2 Research Opportunity. Given this challenge it is prudent to consider what future work should be undertaken to help realise networked distributed sensor systems. One of the first ports of call will be in providing theoretical understanding regarding how these systems will interact when scaled to a simple relay network, 
and what the yardstick should be when we consider system performance. One of the emerging metrics used to measure the success of IoT networks, that provide time relevant information, is the Age of Information (AoI) [18]. By tracking the average time since a successfully arrived packet of information was generated, we are able to infer optimal system design choices. Future research should look to mathematically map these distributed sensor networks and new strategies (i.e. topological design, checkpointing regimes) that enable a minimisation of the average AoI. Computer models should also be developed that map realistic traffic flow on such networks, from which additional insight can be found. This insight should then feed directly into the design choices made by systems researchers.

\subsection{Communication with Non-VLC Platforms on an Intermittent Budget}

3.3.1 Challenge. Communication of the device with non-VLC platforms will also present an interesting challenge for the community. As can be seen in [12], the idea of cross-technological communication (CTC) is already a serious consideration in the field of lowpower VLC. Due to the highly constrained conditions of operation, the deployed technology needs to be very well considered-VLC is useful in some cases, however others may still benefit from radio or a combination of the two (a justification for this in the home environment could be that a VLC uplink is distracting to users, so $\mathrm{RF}$ would be more suitable). The work of [14], much like [12], considers systems that deploy front-end VLC with an uplink radio. It is therefore not unreasonable to think that a VLC IoT system would be integrated as part of a larger network running Wifi, Bluetooth, and other radio based communication media.

Given this likely deployment scenario, research must address the fact that the communication of heterogeneous devices in the IoT is further complicated by the introduction of VLC, and even more so by intermittently operating VLC. Generally we can divide CTC into two branches: those requiring dedicated hardware and those that simply deploy physical layer or packet-level changes [6] The challenge to researchers in intermittently operating VLC will be to provide sufficient hardware and software support to account for frequent system failure and the notably different mediums of transmission.

3.3.2 Research Opportunity. Whilst more research is needed to allow for the seamless integration of VLC into the radio dominated IoT market, one of the clear advantages of deploying VLC is that it does not present the same interference challenges that appear when we consider the interaction of Bluetooth, Zigbee, and Wifi [6] Cross-Technology Interference (CTI) can lead to significant packet loss due to shared spectrum, so the introduction of VLC (at least on the downlink) can have significant benefits to successful packet delivery compared with radio based systems.

The opportunity for researches is to develop the physical layer hardware needed to provide efficient transfer of information from VLC to radio platforms. VLC standardisation and its integration into Wifi systems will increasingly help to promote CTC in the VLC domain. Good integration of VLC with Wifi could mean that the Wifi to Zigbee and Wifi to Bluetooth CTCs already developed in literature can be redeployed to cope with the introduction of VLC packets.

The real opportunity facing researches, regarding this paper, is the inclusion of intermittent operation. Frequent power failure, followed by an unknown off time, will create difficulties for systems when applying previously developed CTC methods. Hence, it is recommended that further research is undertaken to understand the feasibility of current VLC-Wifi CTCs with the inclusion of intermittent power failure.

\subsection{Realising the Vision of Miniaturisation}

3.4.1 Challenge. If we are to once again stoke the fire of Smart Dust, where minute computers interact with their environment through collecting energy and sensing, we need to consider where further accommodations can be made to reduce size and how this will impact the system's performance. Continuing to reduce size to the scale imagined in smart dust for a VLC system will have significant engineering implications. It is true that free-space optical links designed for smart dust normally requires a line-of-sight connection [17]. Forwarding of information also necessitates an energy and physical cost. Designing a system that can both receive and transmit information will impact on our ability to reduce the size of the device, since a minimum amount of power will be needed to support a packet transmission, so a minimum capacity of energy storage is required.

3.4.2 Research Opportunity. There are clear research opportunities spawning from the vision of system miniaturisation, many of which have been explored in work towards smart dust. The removal of a battery is a clear step towards miniaturisation by design, yet a number of additional components also need to be re-assessed, and in fact the idea that batteries limit the development of small energy harvesting devices has been questioned in literature [15]. Using super-capacitors (as seen in [21]) does little to rationalise the initial removal of the battery on grounds of miniaturisation-intermittent operation should be as much a commitment to reducing physical size as it is to the removal of batteries. Hence, it is in the interest of the community to further consider how the size of components in designed systems can be reduced, and to what extent this impacts the stability of the system.

\subsection{Reliability}

3.5.1 Challenge. One question that we must ask is whether energy harvesting intermittently operating computing really provides the necessary framework to successfully sense and transmit information in the given scenarios. Reliability has been one of the main critiques of battery-free systems, and it would be naive to claim that battery-free VLC will have the same functionality as a mainspowered device. The long term market success of low-powered VLC could well depend on producing reliable platforms.

3.5.2 Research Opportunity. The clear research opportunity presented by this however, is the fact that the intermittent computing community has already developed strategies to improve the reliability (both in terms of energy consumption and data throughput) of battery-free systems; so it is clear that these should be tested in the batter-free VLC space. Positioning these systems at the very 
edge of environmental conditions means that energy budgets are constrained. There are situations and system requirements where reliability cannot be ensured-however the inclusion of intermittent checkpointing strategies will continue to further the aim of battery-free VLC and may enable operation in previously inaccessible situations. Future research should analyse the extent to which existing transient operating strategies improve the perceived reliability of battery-free VLC devices.

\subsection{Energy Consumption of System}

3.6.1 Challenge. Intermittently operating systems work on the very fringes of resource constraints and the raison d'être of this field is to create devices that are successfully able to undertake sensing/forwarding of information with minimal interruption due to power failure. Here a clear challenge is to continue to reduce energy consumption through hardware, design, and software strategies. As we have already considered-active VLC is expensive as a form of communication compared to traditional radio, and whilst lowpowered active VLC has been explored in literature, applications should be chosen not because of a need to further the VLC field, but because of a significant engineering advantage.

3.6.2 Research Opportunity. The work of [12] is testament to the fact that uplink VLC is not always required; however some environments, RF sensitive for example, present a clear use case in which VLC supersedes the use of low powered radio. One way in which the challenge may be combated is through further deployment of passive VLC; through modulating LCDs or smart surfaces. The work of [30] presents a comprehensive insight into the numerous challenges in passive VLC, with [19] also deploying passive methods to reduce energy consumption. Evidently then, the hardware research objectives of this paper will be concordant with those already discussed in literature.

In terms of the intermittent software, the work of [3] presents an average $16 \%$ reduction in energy consumption compared with other state of the art transient operating systems-and hence an even greater improvement in energy consumption compared with no strategy. It is quite clear then that porting such checkpointing approaches will provide researchers with additional energy saving opportunities to those previously identified in hardware alone.

\subsection{Proof of Concept Using Existing Technology}

3.7.1 Challenge. A clear challenge for the systems community will be to create a functional battery-free VLC system that deploys computation retention strategies developed in the intermittent computing community.

3.7.2 Research Opportunity. The natural research opportunity here is the continuation of work seen in [21] and [19] to include the strategies developed in works such as [3] and [16]. Further to this, it may also be beneficial to understand how systems such as OpenVLC [29] can be modified to function on harvested energy (and how intermittent operating systems can be applied to them).

\section{WHERE COULD THESE SYSTEMS BE DEPLOYED?}

An intermittently operating VLC device would be able to function at the very limit of computing from the perspective of resource constraints. Hence, the possible number of applications is extensive and can include environments that would traditionally be inaccessible due to limitations of current architecture. We know for example, that VLC has clear applications in RF sensitive environments due to reduced interference and a reduced risk to critical systems. We also know that batteries are particularly liable in extreme temperatures [24], so alternative approaches to energy harvesting and storage would be better suited in such cases.

Space Exploration: A field in which such technology could prove useful is space exploration. Swarms of small space satellites have been proposed in [2] as a possible future for sensing and exploration. Such devices would naturally benefit from energy harvesting architecture due to the limited ability to recharge, and the removal of batteries could improve performance in extreme temperatures whilst also reducing physical size. VLC has been explored in literature as an effective method of communication in small satellites [1,23], so the combination of energy harvesting and VLC would be a natural step to further enhance satellite based sensor networks.

Built Environment: As can be seen in the hardware of [12], ultralow powered VLC system can also be uni-directional and simply receive information via light. Such a device would be ideally suited to small tasks in the built environment. Techniques such as energy disaggregation (which looks to identify the power consumption of devices in buildings through analysis of the total power), while well-versed in literature, may lack sufficient accuracy to provide useful feedback [4]. The development of novel energy submetering devices that utilise battery-free architecture, as hypothesised in [5], could counter issues faced in non-invasive load monitoring by observing each appliance/light individually. Here the device would be able to harvest energy from the environment, and then transmit information about energy consumption or useful building safety measurements.

Agriculture: The agricultural industry could benefit from combined VLC and intermittently operating architecture. The integration of IoT into farming [10] will allow for greater control over environmental conditions, affording more precise action to farmers and thus improving crop yields. Small battery-free energy harvesting devices would be perfectly suited to agriculture due to a reduced ability to hard-wire or replace batteries. Vertical farming would be particularly well suited to such a device, since the environment is more controlled, so line-of-sight communication would be easier to achieve. A device in this scenario may be able to receive information from the lighting infrastructure in the vertical farm (such as frequency of sensing) and then perform small tasks based on this-here the lighting of the crop could also power the device. Passive VLC could also be used to modulate the light used for the plants, and in turn return a useful measurement, such as soil $\mathrm{pH}$ or moisture levels. Transient operating systems would help to make these devices more efficient and more resilient to power failures. 


\section{CONCLUSIONS}

Whilst there is great nobility in creating new fields of work, innovation often occurs by realising that existing technologies can be mutually beneficial. By co-deploying two emerging IoT technologies, intermittent operating strategies and low energy visible light communication, we believe that there is significant potential to improve the performance of existing battery-free devices and develop new systems. VLC based on intermittent computing has clear applications in RF sensitive environments that require small physical devices with little to no maintenance costs. This paper has identified clear use cases in the built environment, agriculture, and space research that we believe should be considered by the systems community.

\section{ACKNOWLEDGEMENTS}

We gratefully acknowledge the financial support of project ENLIGHT'EM (European Training Network in Low-Energy Visible Light IoT Systems, H2020-MSCA-ITN-2018, grant number 814215).

\section{REFERENCES}

[1] David Amanor, William Edmonson, and Fatemeh Afghah. 2018. Inter-Satellite Communication System based on Visible Light. IEEE Trans. Aerospace Electron. Systems 54, 6 (Dec. 2018), 2888-2899.

[2] Justin Atchison and Mason Peck. 2012. A Millimeter-Scale Lorentz-Propelled Spacecraft. In Proc. Guidance, Navigation and Control Conference and Exhibit. AIAA.

[3] Domenico Balsamo, Alex S. Weddell, Anup Das, Alberto Rodriguez Arreola, Davide Brunell, Bashir M. Al-Hashimi, Geoff V. Merrett, and Luca Benini. 2016 Hibernus++: A Self-Calibrating and Adaptive System for Transiently-Powered Embedded Devices. IEEE Transactions on Computer-Aided Design of Integrated Circuits and Systems 35, 12 (2016), 1968-1980.

[4] Nipun Batra, Amarjeet Singh, and Kamin Whitehouse. 2015. If You Measure It, Can You Improve It? Exploring The Value of Energy Disaggregation. In Proc. BuildSys. ACM, 191-200.

[5] Bradford Campbell, Joshua Adkins, and Prabal Dutta. 2016. Cinamin: A Perpetual and Nearly Invisible BLE Beacon. In Proc. EWSN. ACM, 331-332.

[6] Ying Chen, Ming Li, Pengpeng Chen, and Shixiong Xia. 2019. Survey of Crosstechnology Communication for IoT Heterogeneous Devices. IET Communications 13, 12 (2019), 1709-1720.

[7] Cisco. 2020. Cisco Annual Internet Report. Retrieved May 27, 2020 from https:/www.cisco.com/c/en/us/solutions/collateral/executive-perspectives/ annual-internet-report/white-paper-c11-741490.html

[8] Joshua Curry and Nick Harris. 2019. Powering the Environmental Internet of Things. Sensors 19, 8 (April 2019).

[9] Jasper de Winkel, Carlo Delle Donne, Kasim Sinan Yildirim, Przemysław Pawełczak, and Josiah Hester. 2020. Reliable Timekeeping for Intermittent Computing. In Proc. ASPLOS. ACM, 53-67.

[10] Shoaib Farooq, Shamyla Riaz, Adnan Abid, Kamran Abid, and Muhammad Azhar Naeem. 2019. A Survey on the Role of IoT in Agriculture for the Implementation of Smart Farming. IEEE Access 7 (Oct. 2019), 156237-156271.
[11] BeagleBoard.org Foundation. 2019. Beagle Bone Black. Retrieved June 4, 2020 from http://beagleboard.org/black

[12] Ander Galisteo, Ambuj Varshney, and Domenico Giustiniano. 2020. Two to Tango: Hybrid Light and Backscatter Networks for Next Billion Devices. In Proc. MobiSys. ACM.

[13] Josiah Hester and Jacob Sorber. 2017. The Future of Sensing is Batteryless, Intermittent, and Awesome. In Proc. SenSys. ACM.

[14] Muhammad Ismail, Muhammad Zeeshan Shakir, Khalid A. Qaraqe, and Erchin Serpedin. 2016. Radio Frequency and Visible Light Communication Internetworking. Wiley, 119-138.

[15] Neal Jackson, Joshua Adkins, and Prabal Dutta. 2018. Reconsidering Batteries in Energy Harvesting Sensing. In Proc. ENSsys. ACM, 14-18.

[16] Hrishikesh Jayakumar, Arnab Raha, Woo Suk Lee, and Vijay Raghunathan. 2015. QuickRecall: A HW/SW Approach for Computing across Power Cycles in Transiently Powered Computers. F. Emerg. Technol. Comput. Syst. 12, 1 (Aug. 2015).

[17] Joseph M. Kahn, Randy Howard Katz, and Kristofer S. J. Pister. 2000. Emerging Challenges: Mobile Networking for "Smart Dust". Fournal of Communications and Networks 2, 3 (Sept. 2000), 188-196.

[18] Sanjit Kaul, Roy Yates, and Marco Gruteser. 2012. Real-time Status: How Often Should one Update?. In Proc. INFOCOM. IEEE, 2731-2735.

[19] Jiangtao Li, Angli Liu, Guobin Shen, Liqun Li, Chao Sun, and Feng Zhao. 2015. Retro-VLC: Enabling Battery-Free Duplex Visible Light Communication for Mobile and IoT Applications. In Proc. HotMobile. ACM, 21-26.

[20] Angli Liu, Jiangtao Li, Guobin Shen, Chao Sun, Liqun Li, and Feng Zhao. 2018. Enabling Low-power Duplex Visible Light Communication. (2018). arXiv:cs.NI/1801.09812

[21] Xiangyu Liu, Xuetao Wei, Liang Guo, Yejun Liu, Qingyang Song, and Abbas Jamalipour. 2019. Turning the Signal Interference into Benefits: Towards Indoor Self-Powered Visible Light Communication for IoT Devices in Industrial RadioHostile Environments. IEEE Access 7 (Feb. 2019), 24978-24989.

[22] Brandon Lucia, Vignesh Balaji, Alexei Colin, Kiwan Maeng, and Emily Ruppel. 2017. Intermittent Computing: Challenges and Opportunities. In Proc. SNAPL (May 7-10). Alisomar, CA, USA, 8:1-8:14

[23] Zhang Minglun, Yuan Xueguang, and Huang Yong-qing. 2016. A 10.7 km Visible Light Communications Experiment. In Proc. International Conference on Ubiquitous and Future Networks. IEEE, 231-234.

[24] M. R. Palacín and A. de Guibert. 2016. Why do batteries fail? Science 351, 6273 (2016).

[25] Parth H. Pathak, Xiaotao Feng, Pengfei Hu, and Prasant Mohapatra. 2015. Visible Light Communication, Networking, and Sensing: A Survey, Potential and Challenges. IEEE Communications Surveys \& Tutorials 17, 4 (Fourth quarter 2015), 2047-2077.

[26] Saeed Rehman, Shakir Ullah, Peter Jan Joo Chong, Sira Yongchareon, and Dan Komosny. 2019. Visible Light Communication: A System Perspective-Overview and Challenges. Sensors 19 (March 2019).

[27] Stefan Schmid, Giorgio Corbellini, Stefan Mangold, and Thomas R. Gross. 2013. LED-to-LED Visible Light Communication Networks. In Proc. MobiHoc. ACM, $1-10$.

[28] Zhao Tian, Kevin Wright, and Xia Zhou. 2016. The Darklight Rises: Visible Light Communication in the Dark. In Proc. MobiCom. ACM, 495-496.

[29] Qing Wang, Domenico Giustiniano, and Daniele Puccinelli. 2014. OpenVLC: Software-Defined Visible Light Embedded Networks. In Proc. ACM MobiCom Workshop on Visible Light Communication Systems. ACM, 15-20.

[30] Qing Wang and Marco Zuniga. 2017. Passive Sensing and Communication Using Visible Light: Taxonomy, Challenges and Opportunities. (2017). arXiv:cs.NI/1704.01331

[31] Xieyang Xu, Yang Shen, Junrui Yang, Chenren Xu, Guobin Shen, Guojun Chen, and Yunzhe Ni. 2017. PassiveVLC: Enabling Practical Visible Light Backscatter Communication for Battery-Free IoT Applications. In Proc. MobiCom. ACM, 180192. 